\title{
Biological Sciences Tutorial: CryoEM with Phase Plates
}

\author{
Radostin Danev ${ }^{1}$ \\ 1. Max Planck Institute of Biochemistry, Department of Molecular Structural Biology, Martinsried, \\ Germany.
}

Phase plates for transmission electron microscopy (TEM) have been in development for many years but only recently became commercially available. Their main function is improving the image contrast by enhancing the phase contrast performance of the microscope. This is especially beneficial for cryoelectron microscopy (cryoEM) where low image contrast is a typical issue. Phase plates add more complexity to the operation of the microscope and thus require additional training and experience. In this tutorial you will learn about the principle of operation, basic microscope alignments and image processing approaches associated with the use of phase plates.

The most important microscope alignments for proper phase plate operation are the on-plane condition and the beam shift pivot points (Fig. 1). The on-plane condition (Figs. 1a, b) concerns the vertical position of the central beam crossover relative to the phase plate plane. Phase plates operate optimally when the beam crossover is on the phase plate plane, i.e. "on-plane". In suitably designed microscopes the on-plane condition corresponds to parallel illumination on the sample. The beam shift pivot points (Figs. 1c, d) are responsible for the lateral stability of the beam crossover on the phase plate. When the pivot points are properly set the beam crossover remains stationary at a fixed point on the phase plate irrespective of the beam shift movements on the sample. This setting is especially important for cryotomography where beam shifts combined with image shifts are used to keep the area of interest centred throughout the tilt series acquisition.

The Volta phase plate (VPP) [1] consists of a continuous amorphous carbon film and the phase shift is created on-the-fly by the interaction of the central beam with the film. It is relatively easy to use and automate because it does not require precise centering of a phase plate feature, e.g. a hole, on the central beam. In addition to the on-plane and beam shift pivot point alignments, the third important factor for a successful VPP observation is proper conditioning of the phase plate [1,2,3]. The phase shift of the VPP is created by the central beam and builds up gradually with the total dose on the phase plate. A calibration curve, like the blue line in Fig. 2, can be used to calculate the pre-irradiation time required for creating a desired phase shift. The pre-irradiation time in seconds is equal to the dose in $\mathrm{nC}$ (from the horizontal axis in Fig. 2) divided by the total beam current in nA.

Phase plate data is processed with the standard cryoEM software tools and techniques. Single particle data collected with the VPP and a small amount of defocus requires contrast transfer function (CTF) fitting and correction with an additional phase shift parameter [4]. Phase plate cryo-tomography data is usually collected in-focus and does not require CTF correction [5].

\section{References:}

[1] R Danev et al, PNAS 111 (2014) p. 15635.

[2] Y Fukuda et al, Journal of Structural Biology 190 (2015) p. 143.

[3] R Danev and W Baumeister, eLife 5 (2016) doi: 10.7554/eLife.13046.

[4] R Danev, D Tegunov and W Baumeister, eLife 6 (2017) doi: 10.7554/eLife.23006. 
[5] M Khoshouei et al, Journal of Structural Biology 197 (2017) p. 94.

(a)

$$
\text { On-plane }
$$

(parallel illumination)

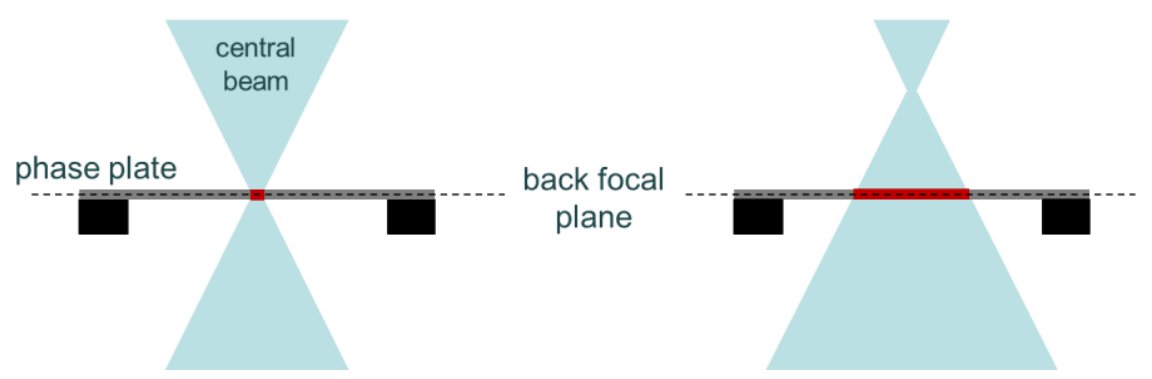

(c) Correct pivot point setting

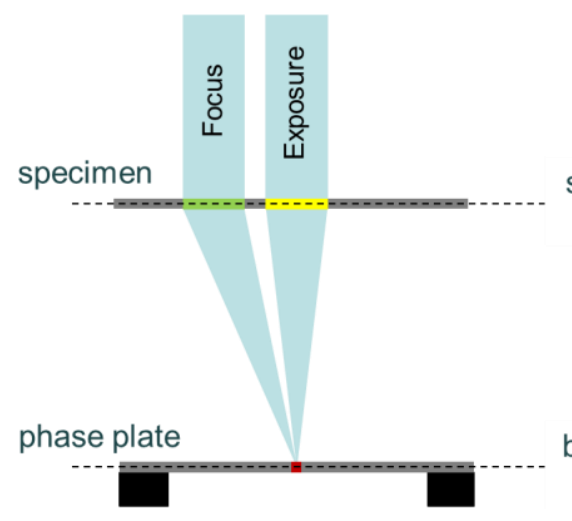

(b)

Off-plane

(non-parallel illumination)

(d) Incorrect pivot point setting

Figure 1. (a, b) On-plane and off-plane conditions. (c, d) Correct and incorrect setting of the beam shift pivot points.

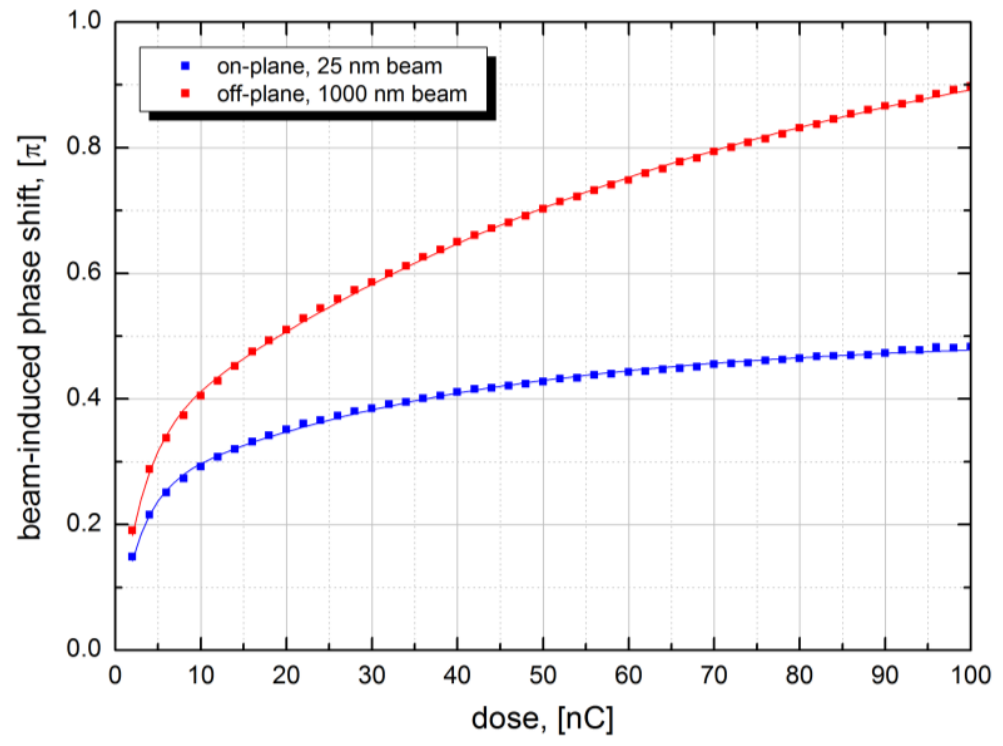

Figure 2. Phase shift vs. dose for the Volta phase plate on-plane (blue curve) and off-plane (red curve). 